\title{
Frequency of etiological causes of intestinal obstruction in children.
}

1. MBBS, FCPS

Senior Registrar Paediatric Surgery National Institute of Child Health, Karachi.

2. MBBS, FCPS

Associate Professor Paediatric Surgery

National Institute of Child Health, Karachi.

3. MBBS, FCPS

Senior Registrar Paediatric Surgery National Institute of Child Health, Karachi.

Correspondence Address:

Dr. Mishraz

Department of Paediatric Surgery

National Institute of Child Health,

Karachi.

mishrazlumhs@hotmail.com

Article received on:

14/10/2020

Accepted for publication:

$12 / 02 / 2021$

\section{Mishraz ${ }^{1}$, Naima Zamir ${ }^{2}$, Muhammad Azhar ${ }^{3}$}

ABSTRACT... Objective: To determine the frequency of the various etiological causes of intestinal obstruction in children. Study Design: Descriptive Cross Sectional Study. Setting: Department of Pediatric Surgery, National Institute of Child Health, Karachi. Period: January 2017 to December 2017. Material \& Methods: Total 143 patients included in study with age range d from 1 month to 12 years. All patients who fulfilled the inclusion criteria and visited to Outpatient/ Emergency department of National Institute of Child health, Karachi were included in the study. Patients followed diagnosis of intestinal obstruction were operated and the outcome variables (Round worms, adhesions, intussusceptions and obstructed hernia) were noted. Data was entered and analyzed through SPSS version: 22. Mean and standard deviation were calculated for age and duration of symptoms. Results: The Mean \pm SD of age was $35.41 \pm 27.664$ months. Out of 143 patients $118(82.5 \%)$ were male and $25(17.5 \%)$ were female. Etiological causes i.e. Adhesive bowel obstruction 59(41.3\%), intussusception 51 (35.7\%), obstructed hernia and worm infestation was found in $23(16.1 \%)$ and 10(7\%) children. Conclusion: It is to be concluded that pattern of intestinal obstruction varies at different age groups in terms of etiology. Adhesion was found to be most common cause of intestinal followed by intussusceptions. However, there is a need to conduct more studies using large sample size with multiple study sites in Pakistan to validate these results.

Key words: $\quad$ Adhesions, Causes, Intussusception, Intestinal Obstruction, Pattern, Round Worms.

Article Citation: Mishraz, Zamir N, Azhar M. Frequency of etiological causes of intestinal obstruction in children. Professional Med J 2021; 28(10):1372-1375. https://doi.org/10.29309/TPMJ/2021.28.10.6146

\section{INTRODUCTION}

Intestinal obstruction is commonly encountered emergency in surgery departments, with considerable morbidity and high mortality. ${ }^{1}$ The presentation of children having intestinal obstruction, have significantly higher chances for complications, proving to be a mighty challenging task, requiring utmost attention and health care facilities especially as in Pediatrics surgery departments having facilities for intensive care. A child presenting with abdominal distention, persistent vomiting, abdominal pain and constipation must be suspected to have an intestinal obstruction. ${ }^{2}$

Most common cause of intestinal obstruction amongst children include hernias, adhesions, worm obstruction, volvulus and

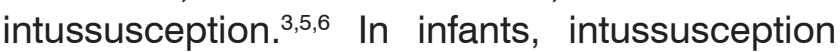

while in older children, worms infestation have been observed to be most commonly reported intestinal obstruction cause. ${ }^{4}$

Obstruction of the intestine is a condition which has the potential of becoming a life threatening condition, If improperly managed or remains undiagnosed, eventually progressing to compromise their blood supply, leading to necrosis of bowel, followed by perforation, sepsis and death. Therefore early diagnosis and promptness in treatment is a necessity. Although there is international literature on intestinal obstruction in children, none has focused comprehensively on children from Pakistan which has different cultural and geographical background. Thereby strategies could be developed to secure all the patients from intestinal obstruction and prompt treatment may lessen the morbidity and mortality. 


\section{MATERIAL \& METHODS}

This cross sectional study using non-probability convenient sampling technique was conducted in department of pediatric surgery at National Institute of Child Health $(\mathrm{NICH})$, Karachi from January 2017 to December 2018 after approval from the Institute's ethical review board (IERB NO:12/2016). Patients age range 1 month to 14 years with either gender, with sign and symptoms of obstruction intestine for at least two days and undergoing surgical intervention. Guardians/ parents willing to provide inform consent. Children in which symptoms resolved on conservative management, like electrolyte imbalances (paralytic ileus), gastroenteritis (inflammation or infection of the stomach or intestines), pancreatitis (inflammation of pancreas).

A thorough history and examination was done and recorded. All patients were managed according to standard protocol. Investigations including plain $\mathrm{X}$ ray abdomen, contrast studies (upper and lower gastrointestinal - as indicated by symptoms / signs) were done as per requirement. Patients following diagnosis of intestinal obstruction were operated and the outcome variables (Round worms, adhesions, intussusceptions, obstructed hernia and volvulus) were noted on Performa.

Data was entered and analyzed through SPSS version: 22. Mean and standard deviation were reported for demographics such as age and symptoms duration. Frequency and percentage was recorded for gender, presenting signs and symptoms (Vomiting, abdominal pain, abdominal distensionandconstipation) and etiological causes (Round worms, adhesions, intussusception and obstructed hernia). Stratification in terms of age, gender, symptoms duration, presenting signs and symptoms was done. Post stratification chi- square test was applied. P- Value $\leq 0.05$ considered as significant. Sampling technique was Non Probability Consecutive Sampling.

\section{RESULTS}

From the total of 143 patients included in the study for assessing frequency of etiological causes of intestinal obstruction in children. Out of $143118(82.5 \%)$ were male and $25(17.5 \%)$ female. Mean age was 35.4 months ( 1 month - 12 years) and duration of symptoms 3.94 days. The vomiting was found to be constant symptom in all patients while abdomen pain and distention was found in $129(90.2 \%)$ and $138(96.5 \%)$ patients. The adhesive bowel obstruction was found to be common etiological cause, includes 59(41.3\%) patient, intussusception on second which includes 51(35.7\%), Obstructed inguinal hernia and worm infestation was found in 23 (16.1\%) and $10(7 \%)$ as shown in Table-I and II.

\begin{tabular}{|l|c|}
\hline \multicolumn{1}{|c|}{ Clinical Features } & Number of Patients(n) \\
\hline Vomiting & $143(100 \%)$ \\
\hline Abdominal pain & $129(90.2 \%)$ \\
\hline Abdominal distention & $138(96.5 \%)$ \\
\hline Constipation & $84(58.7 \%)$ \\
\hline \multicolumn{1}{|c|}{ Table-I. Clinical presentation. } \\
\hline \multicolumn{1}{|c|}{ Etiological Causes } & Number of Patients (n) \\
\hline Adhesive obstruction & $59(41.3 \%)$ \\
\hline Round worms & $10(7 \%)$ \\
\hline Intussusception & $51(35.7 \%)$ \\
\hline Obstructed inguinal hernia & $23(16.1)$ \\
\hline \multicolumn{2}{|c|}{ Table-II. Causes of intestinal obstruction. } \\
\hline
\end{tabular}

\section{DISCUSSION}

Intestinal obstruction is the most common pediatric surgery emergency. There are different causes depending upon age. The peak incidence is in infants and elder children. There are also regional variations in etiology. In our research, most commonly reported reason was adhesive bowel obstruction.

Adhesions are the most frequently observed reason for obstruction of intestines amongst infants. In this study, 118 (82.5\%) male and 25 $(17.5 \%)$ were female with male to female ratio $1: 4.72$ which is at variance with other studies. ${ }^{4}$ The fact that in our study, older aged children reported with intestinal obstruction were limited, probably due to the fact that they were treated or managed locally by general surgeons and not referred to Pediatric Surgery department for treatment. Most of the adhesion which causes obstruction is inflammatory in nature. 
Intussusception remained most common cause in other studies ${ }^{2,4,6}$ but in the current study it was second most common cause. In our series of 51 patients, all patients underwent surgical exploration and most of them were idiopathic whilst lead point was reported in only 2 patients. Even then the frequency is higher than that reported in the west, where barium of air reduction is performed and now in few third world populations beginning to perform it as well. ${ }^{6,7,8}$

In a study of Shiekh KA and et al, most frequent etiological reason of intestinal obstruction among children seen was worms $(63.2 \%)$ followed by adhesions (11.1\%), while in our study worm infestation was least commonly observed reason for obstruction of intestine. ${ }^{9}$

Mean duration of symptoms of all the enrolled children was $3.94 \pm 2.645$ days. The median duration was 4 days. In this study age, gender, duration of disease, duration of symptoms, sing and symptoms i.e. (vomiting, abdominal pain, abdominal distension and constipation) played a role of effect modifiers/confounders. It was found significant association between age group (20--30) and > 36 months and etiological causes. Significant association was found between adhesions, intussusceptions and obstructed hernia with gender and no association was found with round worms. Similar to the above findings, our study also reported results in line with them.

Although in this study, cause of obstruction and demographics were reported, however the study was not immune from observer and selection bias and the fact that the study was carried out at a single center having a very limited sample size,. Using non-probability sampling technique also limited generality. Therefore further multicentered studies having greater sample size and other demographics would be enlightening in determining better results regard intestinal obstruction. On the contrary, strength of the study includes study design and sample selection due to strict inclusion and exclusion criterion. Using predictor definitions and variables of outcomes minimized source of bias.

\section{CONCLUSION}

It is to be concluded that reported frequency of intestinal obstruction varied among the age groups with regards to the etiological factors and their relative incidence. Adhesion was found to be most common etiological cause of intestinal followed by intussusceptions. Furthermore; manifestation of signs and symptoms depend highly on the level of obstruction. However, outcome was not affected by the cause of obstruction only.

Copyright $@ 12$ Feb, 2021.

\section{REFERENCES}

1. Paulson EK, Thompson WM. Review of small-bowel obstruction: The diagnosis and when to worry. Radiology. 2015 May; 275(2):332-42.

2. Bhedi A, Prajapati M, Sarkar A. A prospective study of intestinal obstruction in paediatric age group. International Surgery Journal. 2017 May 24; 4(6):197983.

3. Sathyanarayana SA, Deutsch GB, Friedman B. An interesting cause of mechanical small bowel obstruction. Indian Journal of Surgery. 2015 Dec 1; $77(2): 731-2$.

4. Baiu I, Hawn MT. Small bowel obstruction. Jama. 2018 May 22; 319(20):2146-.

5. Houben $\mathrm{CH}$, Pang KK, Mou WC, Chan KW, Tam YH, Lee $\mathrm{KH}$. Epidemiology of small-bowel obstruction beyond the neonatal period. Annals of Pediatric Surgery. 2016; 12(3):90-3.

6. Gfroerer S, Rolle U. Pediatric intestinal motility disorders. World Journal of Gastroenterology: WJG. 2015 Sep 7; $21(33)$ :9683-7.

7. Mansoor H, Yousaf UF. Intussusception: What leads in children beyond infancy? Ann King Edward Med Coll 1998; 4:31-2.

8. Jackson P, Cruz MV. Intestinal obstruction: Evaluation and management. American family physician. 2018 Sep 15; 98(6):362-7.

9. Shiekh KA, Baba AA, Ahmad SM, Shera AH, Patnaik $R$, Sherwani AY. Mechanical small bowel obstruction in children at tertiary care centre in Kashmir. Afr $\mathrm{J}$ Paediatr Surg. 2010; 7(2):81-5. 


\begin{tabular}{|c|c|c|c|}
\hline \multicolumn{4}{|c|}{ AUTHORSHIP AND CONTRIBUTION DECLARATION } \\
\hline Sr. \# & Author(s) Full Name & Contribution to the paper & Author(s) Signature \\
\hline 1 & & $\begin{array}{l}\text { Conception, Write up, } \\
\text { Analysis and design of study. }\end{array}$ & Mishraz Shaikh \\
\hline 2 & Naima Zamir & $\begin{array}{l}\text { Conception and design of } \\
\text { study. }\end{array}$ & $\begin{array}{l}\text { Naima zamir } \\
\text { Ozhar MII }\end{array}$ \\
\hline
\end{tabular}

